\title{
Ulzerationen unter Therapie mit Hydroxycarbamid
}

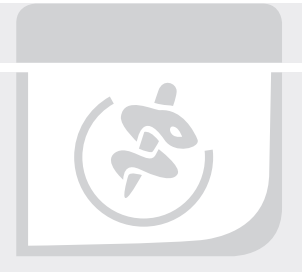

\section{Fallbericht}

Ein 73-jähriger Patient stellte sich zur operativen Therapie epithelialer Tumoren im Gesichtsbereich vor. Nebenbefundlich zeigten sich im Knöchelbereich beidseits und an der lateralen rechten Fußkante unterschiedlich große, teils bizarr geformte, fibrinös belegte Ulzera mit einer Atropie blanche ( $\mathbf{A b b . 1 , ~}>$ Abb. 2]. Diese Ulzerationen bestanden anamnestisch seit einem Jahr. Seit 14 Jahren war ein myeloproliferatives Syndrom mit Polyzythämia vera bekannt. Diese war in den beiden vorangehenden Jahren mit Hydroxycarbamid (Litalir $\left.{ }^{\circledR}\right) 500 \mathrm{mg} / \mathrm{d}$ behandelt worden. Bei zunehmendem Hämatokrit war die Dosis nach 6 Monaten Behandlungsdauer auf Hydroxycarbamid $1000 \mathrm{mg} / \mathrm{d}$ erhöht worden. Unter Hydroxycarbamid 1000 mg/d, oraler Antikoagulation mit Apixaban 5 mg zweimal täglich und regelmäßigen Aderlässen zeigte sich die Polyzythämia vera klinisch gut kontrolliert. Wir führten im Rahmen des stationären Aufenthaltes des Patienten neben einer stadiengerechten Wundtherapie eine sechstägige Therapie mit Tinzaparin-Na (Innohep $\left.{ }^{\circledR}\right) 3500$ I. E. subkutan einmal täglich zur Verbesserung der Rheologie durch. Eine chronische venöse Insuffizienz wurde duplexsonographisch ausgeschlossen. Ein Anhalt für eine periphere arterielle Verschlusskrankheit zeigte sich nicht. Trotz Fortführung der lokaltherapeutischen Maßnahmen zeigten die Ulzerationen in den Folgemonaten einen therapierefraktären Verlauf, wobei einige Ulzerationen spontan abheilten und neue an anderer Lokalisation entstanden ( Abb. 3). Aufgrund einer Zunahme der Leuko- und Thrombozytose wurde die Hydroxycarbamid-Therapie nach dreijähriger Behandlungsdauer durch den betreuenden Hämatoonkologen umgestellt auf pegyliertes Interferon alpha 2b (PegIntron ${ }^{\circledR}$ ). Einen Monat nach Beendigung der Therapie mit Hydroxycarbamid zeigten sich die Ulzerationen vollständig abgeheilt.

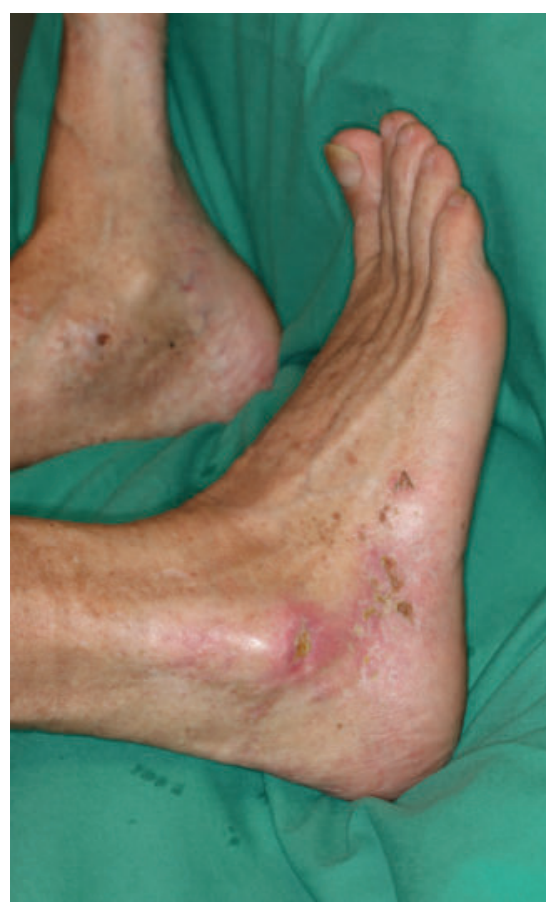

-Abb. 1 Ulzeration im Knöchelbereich unter Therapie mit Hydroxycarbamid (Litalir $^{\circledR}$ ) (Zeitpunkt 1)

\section{Hintergrund}

\section{Myeloproliferatives Syndrom, Polyzythämia vera}

Der Begriff myeloproliferatives Syndrom bezeichnet eine Gruppe von malignen Erkrankungen, die durch Proliferation einer hämatopoetischen Stammzelle gekennzeichnet sind [1]. Hierzu gehören neben der Polycythaemia vera auch die chronisch myeloische Leukämie, die essentielle Thrombozythämie und die primäre Myelofibrose. Die Therapie richtet sich nach der jeweiligen Form des myeloproliferativen Syndroms und gestaltet sich häufig schwierig. Bei unserem Patienten lag ein myeloproliferatives Syndrom mit Polycythaemia vera vor, bei der die Anzahl der Erythrozyten und somit auch der Hämoglobinwert stark erhöht sind. Die häufigste Komplikation sind thromboembolische Ereignisse (arterielle oder venöse Thromboembolien). Die Therapie der proliferativen Phase der Erkran-

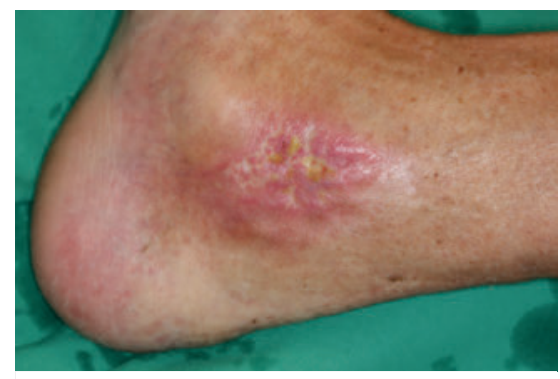

Abb. 2 Detailaufnahme Innenknöchel (Zeitpunkt 1)

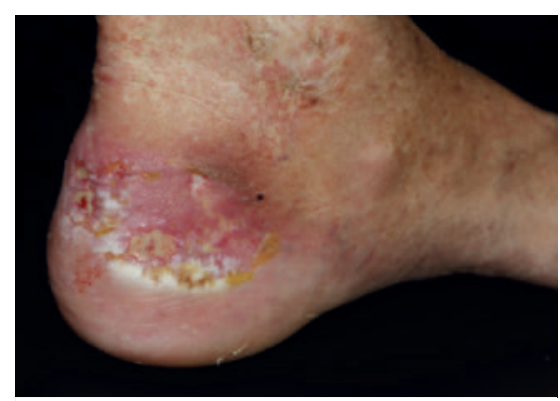

-Abb. 3 Ulzerationen ca. 1 Jahr später (Zeitpunkt 2)

kung besteht in Aderlässen, Antikoagulation und zytoreduktiver Chemotherapie. Bringen regelmäßige Aderlässe nicht den gewünschten Erfolg, so erfolgt die Therapieeskalation mit Hydroxycarbamid. Medikamente der zweiten Wahl sind neben pegyliertem Interferon alpha der Tyrosinkinaseinhibitor Ruxolitinib und das Alkylans Busulfan [1].

\section{Wie entstehen Ulzera unter Hydroxycarbamid-Therapie?}

Hydroxycarbamid oder Hydroxyurea (Handelsnamen: Litalir ${ }^{\circledR}$, Syrea ${ }^{\circledR}$ ) ist ein zytotoxisches Chemotherapeutikum. Dieses wird seit den 1960er-Jahren bei myeloproliferativen Erkrankungen angewendet. Durch die Hemmung der Ribonukleotidreduktase wird Reduktion von Ribose zu Desoxyribose verhindert. Hierdurch wird direkt die DNA-Synthese blockiert [2]. Neben der gewünschten Wirkung mit Hemmung der Zellteilung bei hämatogenen Neoplasien kommt es zu Nebenwirkungen wie 
Schleimhauterosionen und Transaminasenerhöhung. Aufgrund des Wirkmechanismus ist auch ein karzinogenes Risiko denkbar. Die Datenlage hierzu ist bislang uneinheitlich [2, 3]. Dermatologische Nebenwirkungen durch Hydroxyurea sind gut bekannt. Diese reichen von Erythemen, Hyperpigmentierungen, Schuppung und Atrophie der Haut, Alopezie, Nagelwachstumsstörungen und Hauttumoren bis zum Auftreten von schmerzhaften Ulzera insbesondere im Bereich der Malleoli [4].

Die Häufigkeit von Ulzera unter Therapie mit Hydroxycarbamid wird in der Literatur mit 8-9\% der behandelten Patienten angegeben $[2,5]$, in den Fachinformationen je nach Hersteller mit „sehr häufig“ (>10\%) bis „häufig“ (1-10\%). Insgesamt gilt es zu berücksichtigen, dass auch hämatoonkologische Grunderkrankungen selbst zu Hautulzerationen führen können $[4,6]$.

Die Entstehung der Ulzerationen wird zum einen mit der Entstehung einer Megaloblastose (große, unreife Erythrozyten) bei längerer Einnahme von Hydroxycarbamid erklärt. Diese führt zu einer Hyperviskosität der Erythrozyten und somit zu einer Beeinträchtigung der Mikrozirkulation der Haut $[5,6]$. Zu dieser Theorie passt die mikroangiopathische Morphologie der Hydroxycarbamid-induzierten Ulzera. Zum anderen wirkt Hydroxycarbamid direkt zytotoxisch auf die basalen Keratinozyten und auf Kollagenfasern [5, 6], so dass die Haut verletzlicher ist und leichter exulzeriert.

Die Ulzera können zu jedem Zeitpunkt unter der Therapie mit Hydroxycarbamid auftreten. Im Durchschnitt kommt es drei Jahre nach Beginn der Therapie zum Auftreten von Ulzerationen [3]. Die Höhe der Hydroxycarbamid-Dosis korreliert mit der
Häufigkeit und der Schwere der Ulzerationen. Nach Absetzen von Hydroxycarbamid kommt es in der Regel zur raschen Abheilung innerhalb weniger Monate. Aufgrund der zugrundeliegenden, prognosebestimmenden hämatoonkologischen Grunderkrankungen ist ein Absetzen von Hydroxyurea oftmals nicht oder nur kurzfristig möglich. In diesen Fällen empfiehlt sich umso mehr die konsequente und stadienadaptierte Wundtherapie [4].

Interessenkonflikt

Die Autoren geben an, dass kein Interessenkonflikt besteht.

\section{Autorinnen/Autoren}

\section{K. Neumann}

A. L. Recke

Institute

Klinik für Dermatologie, Allergologie und Venerologie, UKSH Schleswig-Holstein, Campus Lübeck

\section{Korrespondenzadresse}

\section{Dr. Anna Lena Recke}

Klinik für Dermatologie, Venerologie und Allergologie

UKSH Campus Lübeck

Ratzeburger Allee 160

23538 Lübeck

E-Mail: Anna.Recke@uksh.de
Literatur

[1] Tefferi A, Vardiman JW. Myelodysplastic syndromes. N Engl J Med. 2009; 361(19): 1872-1885

[2] Fram RJ, Kufe DW. Inhibition of DNA excision repair and the repair of $x$-ray induced DNA damage by cytosine arabinoside and hydroxyurea. Pharmacol Ther 1985; 31(3): 165-176

[3] Boneberger S, Rupec RA, Ruzicka T. Ulcers following therapy with hydroxyurea. Three case reports and review of the literature. Hautarzt 2010; 61(7):598-602

[4] Soutou B, Aractingi S. Myeloproliferative disorder therapy: assessment and management of adverse events - a dermatologist's perspective. Hematol Oncol 2009; 27 Suppl 1: 11-3

[5] Hwang SW, Hong SK, Kim SH et al. A Hydroxyurea-induced Leg Ulcer. Ann Dermatol 2009; 21(1): 39-41

[6] Knupfer J, Stücker M, Reich-Schupke S. Differenzialdiagnosen von Ulzerationen an Bein und Fuß. Aktuelle Dermatologie 2018; 04: $164-179$

Bibliografie

DOI https://doi.org/10.1055/a-0830-0831 Phlebologie 2019; 48: 16-17 (c) Georg Thieme Verlag KG Stuttgart · New York ISSN 0939-978X 